2013-1

\title{
Effect Of Glycerol On A Diacetone Acrylamide-Based Holographic Photopolymer Material
}

\author{
Dervil Cody \\ Technological University Dublin, dervil.cody@tudublin.ie \\ Izabela Naydenova \\ Technological University of Dublin, izabela.naydenova@tudublin.ie \\ Emilia Mihaylova \\ Technological University of Dublin, emilia.mihaylova@tudublin.ie
}

Follow this and additional works at: https://arrow.tudublin.ie/cieoart

\section{Recommended Citation}

Cody, D., Naydenova, I. \& Mihaylova, E. (2013) Effect of glycerol on a diacetone acrylamide-based holographic photopolymer material, Applied Optics 52(3), 489-494. doi:10.1117/12.923116

This Article is brought to you for free and open access by the Centre for Industrial and Engineering Optics at ARROW@TU Dublin. It has been accepted for inclusion in Articles by an authorized administrator of ARROW@TU

Dublin. For more information, please contact

arrow.admin@tudublin.ie, aisling.coyne@tudublin.ie, gerard.connolly@tudublin.ie.

Funder: Irish Research Council

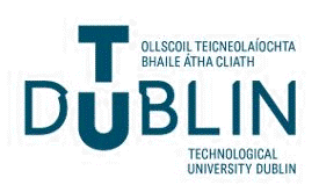




\title{
Effect of glycerol on a diacetone acrylamide-based holographic photopolymer material
}

\author{
Dervil Cody, ${ }^{1,2}$ Izabela Naydenova, ${ }^{1,2}$ and Emilia Mihaylova ${ }^{1,2,3, *}$ \\ ${ }^{1}$ Centre for Industrial and Engineering Optics, Dublin Institute of Technology, Dublin 8, Ireland \\ ${ }^{2}$ School of Physics, College of Sciences and Health, Dublin Institute of Technology, Dublin 8, \\ Ireland \\ ${ }^{3}$ Department of Mathematics and Physics, Agricultural University, Plovdiv, Bulgaria \\ *Corresponding author: emilia.mihaylova@dit.ie
}

The composition of the low toxicity, environmentally-compatible Diacetone Acrylamide photopolymer has been modified with the inclusion of different additives. The addition of glycerol to the photopolymer composition is described. Results show that the incorporation of glycerol results in a uniform maximum refractive index modulation for recording intensities in the range of $1-20 \mathrm{~mW} / \mathrm{cm}^{2}$. This may be attributed to glycerol's nature as a plasticizer, which allows for faster diffusion of un-reacted monomer within the grating during holographic recording. An optimum recording intensity of $0.5 \mathrm{~mW} / \mathrm{cm}^{2}$ is observed for exposure energies of $20-60 \mathrm{~mW} / \mathrm{cm}^{2}$. The modified photopolymer achieves a refractive index modulation of $2.2 \times 10^{-3}$, with diffraction efficiencies up to $90 \%$ in $100 \mu \mathrm{m}$ layers. Glycerol has also shown to reduce the rate of photobleaching of the diacetone acrylamide photopolymer. This is possibly due to more prevalent inhibition effects caused by increased oxygenation of the photopolymer layers. The stability of the photopolymer samples is also improved with the addition of glycerol.

OCIS codes: $090.0090,160.0160$.

\section{Introduction}

The optimization and characterization of a new non-toxic Diacetone Acrylamide (DA) based photopolymer has previously been described [1]. The improved photopolymer composition is shown to have holographic recording features similar to that of the standard Acrylamide (AA) based photopolymer [2], but with reduced toxicity due to the replacement low-toxicity monomer Diacetone Acrylamide [3-9]. It has also shown to surpass the holographic recording capabilities of other recently developed low toxicity photopolymer materials [10-12]. This materials non-toxic nature, wide dynamic range and high holographic sensitivity, together with its self-processing nature and low cost of production, make it an excellent candidate for applications such as holographic sensors, diffractive optics and in data storage.

Since the DA molecules are larger in size than the AA molecules, one would expect a slower diffusion of the monomer molecules in the DA based photopolymer system. This will be disadvantageous in particular at lower spatial frequencies of recording, where the distance over 
which the mass transport takes place is increased. In order to facilitate monomer diffusion the inclusion of a plasticizer such as glycerol was considered.

This paper details the effect of the addition of glycerol on the holographic recording properties of the new DA photopolymer. Glycerol is a transparent, viscous liquid which is reported to have low-toxicity [13]. It is widely used in the food and pharmaceutical industries as a solvent and lubricant. Glycerol has been shown to influence the maximum refractive index modulation's dependence on recording intensity, the rate of photobleaching, and also the stability of the DA photopolymer samples.

\section{Theory}

A typical photopolymer which reacts via a radical chain mechanism consists of a main monomer, a cross-linking monomer, an electron donor, a photosensitizing dye, and a binder matrix, which holds all of the components together. These photopolymer components are spatially redistributed during holographic recording due to the polymerization reaction which occurs in the material on exposure to light. This reaction involves three steps: initiation, propagation and termination [14]. When illuminated, dye molecules absorb photons of light, and are promoted to excited singlet states.

$$
X D+h v \rightarrow{ }^{1} X D \bullet
$$

These singlet states can re-emit this energy via fluorescence, or by radiationless energy transfer to another molecule.

$$
\begin{gathered}
{ }^{1} X D \bullet \rightarrow X D+h v \\
{ }^{1} X D \bullet+Y \rightarrow X D+Y^{*}
\end{gathered}
$$

Alternatively, the singlet state can be converted to the more stable and longer lived excited triplet state dye molecule, via intersystem crossing.

$$
{ }^{1} X D \bullet \rightarrow{ }^{3} X D \bullet
$$

This triplet state dye molecule then reacts with the electron donor to produce a pair of radicals.

$$
{ }^{3} X D \bullet+E D \rightarrow X D+E D \bullet
$$

Each of these radicals will react with a monomer molecule to produce an initiating species.

$$
E D \bullet+M \rightarrow E D-M \bullet
$$

The growing chain continues to add more monomer units via propagation.

$$
E D-M \bullet+M \rightarrow E D(M)_{2} \bullet
$$

This propagation step will continue until one of two termination reactions occurs, namely combination or disproportionation.

Oxygen quenching is an important process. It causes a reduction in the yield of singlet and triplet state dye molecules, as they are deactivated by any oxygen present in the layer:

$$
\begin{aligned}
& { }^{3} \mathrm{XD} \bullet+{ }^{3} \mathrm{O}_{2} \rightarrow \mathrm{XD}+{ }^{3} \mathrm{O}_{2} \bullet \\
& { }^{1} \mathrm{XD} \bullet+{ }^{3} \mathrm{O}_{2} \rightarrow \mathrm{XD}+{ }^{1} \mathrm{O}_{2} \bullet
\end{aligned}
$$

This can cause an inhibition period at the start of polymerisation. Most of the oxygen in the layer must be used up during holographic exposure before polymerisation can begin $[15,16]$. Glycerol is a known reducing agent and has been shown to stimulate the level of oxygenation in different materials $[17,18]$. The effect of glycerol on photosensitive systems has been studied. Meyer et al. [19] and Galassi [20] report that increasing the concentration of glycerol causes up 
to an order of magnitude drop in the photobleaching rate for two very different photosensitive systems. Slower rates of photobleaching are desirable for holographic photopolymers, as less dye molecules are bleached initially, allowing for longer exposure times before saturation is reached. This allows for the formation of longer polymer chains and hence a higher refractive index modulation. Glycerol is reported to have a pronounced effect on the sensitivity of photopolymer systems also [18].

Here, $1 \mathrm{ml}$ of glycerol solution was added to the DA-based photopolymer composition [1], as optimum layer quality was observed for this concentration. The modified photopolymer has been characterised in terms of its intensity dependence, exposure energy dependence and spatial frequency dependence. The effect of glycerol on the material's rate of photobleaching has been reported also. The modified composition has been compared with the original diacetone acrylamide composition, and the standard acrylamide composition.

Gratings were recorded in $100 \pm 10 \mu \mathrm{m}$ thick layers at spatial frequencies of 1000 and $3000 \mathrm{l} / \mathrm{mm}$. These parameters allow for application of Kogelnik's coupled-wave theory for volume, thick gratings [21], which is used here to determine refractive index modulation $(\Delta n)$ of the recorded gratings:

$$
\Delta n=\frac{\lambda \cos \theta \sin ^{-1}(\sqrt{\eta})}{\pi d}
$$

where $\theta, \lambda$ are the Bragg angle and wavelength of the reconstructing beam. $\eta$ is the diffraction efficiency of the recorded grating (defined here as the ratio of the intensity of the first diffraction order and the incident intensity of the probe beam), and $d$ is the thickness of the photosensitive layer.

\section{Experiment}

\section{A. Preparation of Photopolymer Layers}

The different photopolymer compositions tested were prepared as described in Table 1 . The photopolymer solution was then deposited on to glass slides $(76 \times 26 \mathrm{~mm})$ and allowed to dry for 12-24 hours in darkness under normal laboratory conditions (20-25 ㅇ, 40-60\% RH). Layer thickness was measured using a white-light surface profiler (Micro XAM S/N 8038).

Table 1: Composition of Diacetone Acrylamide and Acrylamide Solutions

\begin{tabular}{llll}
\hline & DAO & DAG & AA \\
\hline PVA 10\% wt/vol (ml) & 20 & 20 & 18 \\
TEA (ml) & 2 & 2 & 2 \\
Diacetone Acrylamide (g) & 1 & 1 & - \\
Acrylamide (g) & - & - & 0.6 \\
Bisacrylamide (g) & 0.2 & 0.2 & 0.2 \\
Erythrosin B 0.11\% wt/vol & 4 & 4 & 4 \\
(ml) & & & \\
Glycerol (ml) & - & 1 & - \\
\hline
\end{tabular}




\section{B. Experimental Set-up}

A two-beam holographic optical setup (see fig.1) with an angle of 30.85 o between the beams was used to record unslanted transmission gratings using a $532 \mathrm{~nm}$ vertically-polarised $\mathrm{Nd}: \mathrm{YVO}_{4}$ laser. Gratings were recorded in the layers with exposure energies of $20-100 \mathrm{~mJ} / \mathrm{cm}^{2}$ at a spatial frequency of $1000 \pm 10$ lines $/ \mathrm{mm}$. The absorption of the photopolymer at $633 \mathrm{~nm}$ is negligible, so a $633 \mathrm{~nm}$ He-Ne laser was used as the probe beam at the Bragg angle. As the recorded gratings are isotropic in nature, their diffraction efficiency is not dependent on the polarisation of the probe beam. An optical power meter (Newport 1830-C) was used to record the intensity of the diffracted beam and LabVIEW software was used to plot the data in real-time. In order to measure the diffracted intensity dependence on the incident angle of the probe beam, the grating was placed on a rotational stage (Newport ESP 300). The accuracy of this measurement was $\pm 1 \times 10^{-3} \mathrm{deg}$.

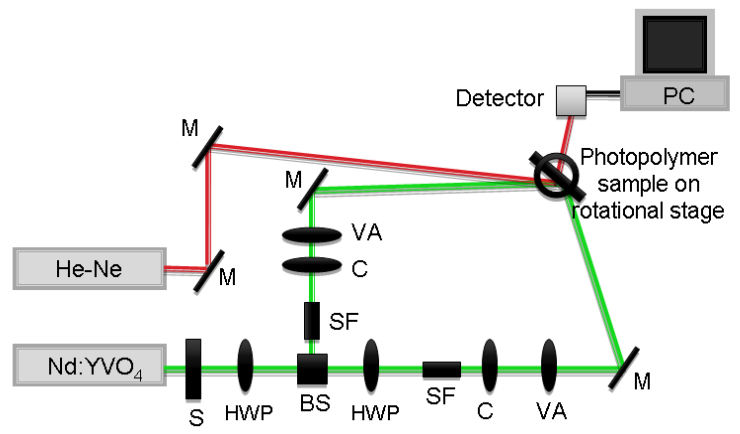

Fig. 1: Experimental setup: S: shutter, HWP: half wave plate, BS: polarising beam splitter, SF: spatial filter, C: collimator, VA: variable aperture, M: mirror.

\section{Results and Discussion}

\section{A. Dependence of Refractive Index Modulation on Recording Intensity}

The intensity dependence of the modified photopolymer at $1000 \mathrm{l} / \mathrm{mm}$ was investigated. Transmission gratings were recorded in photopolymer layers using a recording intensity which was varied from 1-5 $\mathrm{mW} / \mathrm{cm}^{2}$, with constant exposure energy of $100 \mathrm{~mJ} / \mathrm{cm}^{2}$.

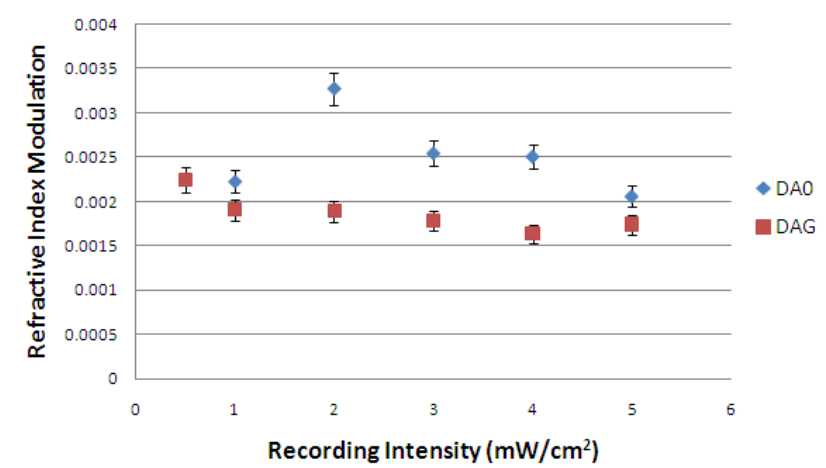

Fig. 2: Refractive index modulation vs. recording intensity for the DAO (no glycerol), DAG (with glycerol) compositions at $1000 \mathrm{l} / \mathrm{mm}$ for an exposure of $100 \mathrm{~mJ} / \mathrm{cm}^{2}$. 
As can be seen from fig. 2, while DA0 (no glycerol) has an obvious optimum intensity at $2 \mathrm{~mW} / \mathrm{cm}^{2}$, the intensity dependence for DAG (with glycerol) is approximately uniform for the range of intensities tested. This is due to glycerol's nature as a plasticizer, which allows the unreacted monomer to diffuse more quickly into the illuminated regions within the layer. For the composition DA0 which contains no glycerol, there is more obvious intensity dependence as the monomer moves slower.

\section{B. Dependence of Refractive Index Modulation on Exposure Energy}

As is shown in fig. 3, DAG reaches an optimum refractive index modulation at $0.5 \mathrm{~mW} / \mathrm{cm}^{2}$, for the lower exposure energies $\left(20-60 \mathrm{~mJ} / \mathrm{cm}^{2}\right)$. This is not as prominent at the higher exposure energies of $80-100 \mathrm{~mJ} / \mathrm{cm}^{2}$. This can be explained by considering the key factor that contributes to grating formation; the ratio of the rate of polymerisation to the rate of monomer diffusion [22].

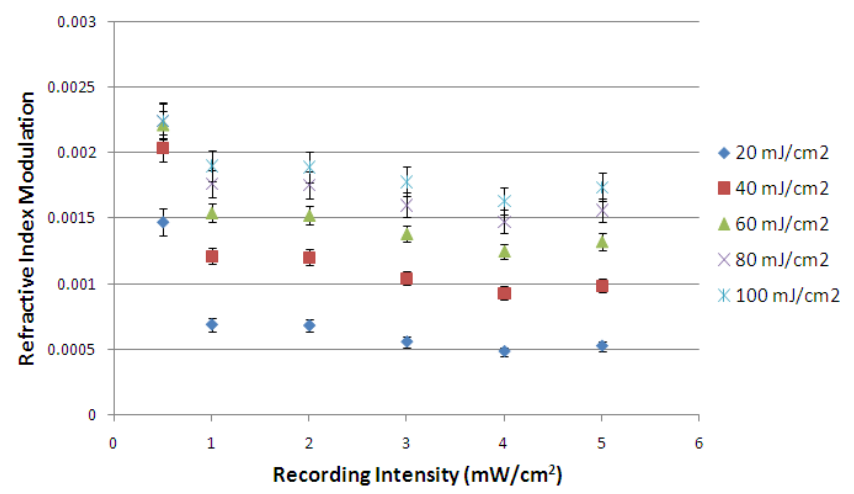

Fig. 3: Refractive index modulation vs. recording intensity for DAG over a range of exposure energies.

At lower recording intensities, the rate of polymerisation is slow due to the low number of initiating species. Therefore the increased rate of diffusion due to the addition of glycerol ensures that the maximum refractive index modulation is reached quickly, as the un-reacted monomer molecules can easily diffuse into the illuminated areas and be polymerised. This explains the trend shown in fig.3. The refractive index modulation reaches a maximum at the lowest recording intensity tested $0.5 \mathrm{~mW} / \mathrm{cm}^{2}$. As the intensity is increased beyond $0.5 \mathrm{~mW} / \mathrm{cm}^{2}$, the rate of polymerization is increased due to a higher concentration of initiating species. Any improvement in the rate of diffusion due to the addition of glycerol is overridden by this increase in the polymerization rate. This results in a decrease in refractive index modulation of $60.12 \%$ for an exposure energy of $20 \mathrm{~mJ} / \mathrm{cm}^{2}$, and $20.24 \%$ for $100 \mathrm{~mJ} / \mathrm{cm}^{2}$, as the recording intensity is increased above $0.5 \mathrm{~mW} / \mathrm{cm}^{2}$.

Higher recording intensities of 10 and $20 \mathrm{~mW} / \mathrm{cm}^{2}$ were then investigated, and the results of this are shown in fig.4. It is observed that there is little difference in the refractive index modulation achieved using recording intensities of 2 or $20 \mathrm{~mW} / \mathrm{cm}^{2}$ over a range of exposure energies. The uniform intensity response of the diacetone acrylamide material is a desirable feature for holographic applications. The use of high recording intensities allows for much faster recording, which is necessary for hologram production on a large scale. 


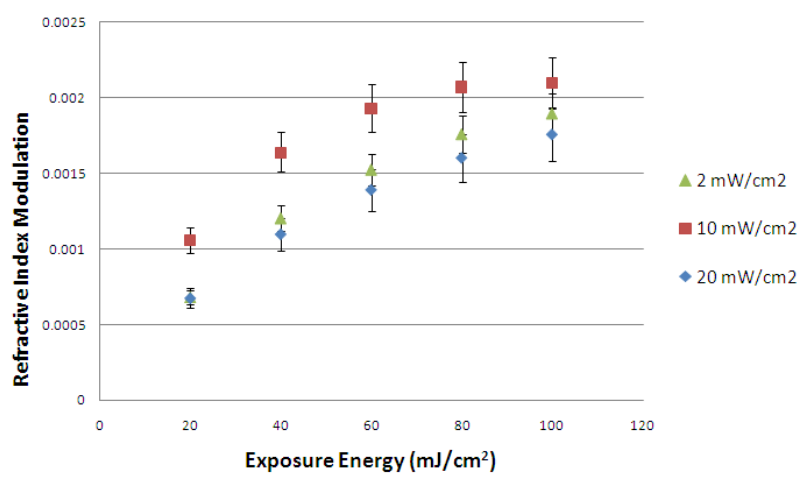

Fig. 4: Refractive index modulation vs. exposure energy for DAG samples tested at low $\left(2 \mathrm{~mW} / \mathrm{cm}^{2}\right)$ and high (10 and $20 \mathrm{~mW} / \mathrm{cm}^{2}$ ) intensities.

\section{C. Dependence of Refractive Index Modulation on Spatial Frequency}

The maximum refractive index modulation for different recording intensities was investigated for the DAG photopolymer at $3000 \mathrm{I} / \mathrm{mm}$. The result of this is shown in fig. 5 . At the higher spatial frequency of $3000 \mathrm{l} / \mathrm{mm}$, the maximum refractive index modulation achievable for DAG falls off, as is also the case for DAO. DAG reaches a maximum RIM of $\sim 3.5 \times 10^{-4}$, which is slightly lower than that achieved with the DAO composition. However DAG retains its linear intensity dependence at the range of recording intensities tested.

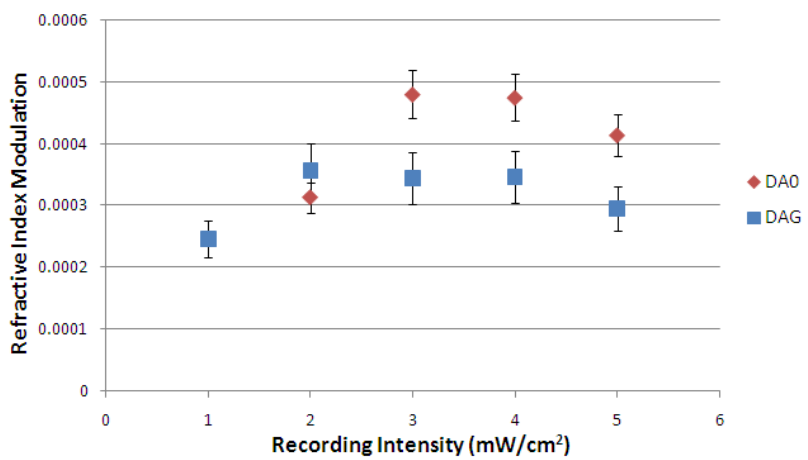

Fig. 5: Refractive Index Modulation vs. recording Intensity for the DAO (no glycerol), DAG (with glycerol) compositions at $3000 \mathrm{l} / \mathrm{mm}$ for exposure energy of $100 \mathrm{~mJ} / \mathrm{cm}^{2}$.

\section{D. Investigation of Inhibition Period due to Glycerol}

An inhibition period was observed at the start of recording for a proportion of the photopolymer layers containing glycerol. This delay is caused by oxygen-quenching in the layer, a process outlined in section 2. In fig. 6 the relationship between the duration of the inhibition period and the recording intensity for DAG is shown. As the recording intensity is increased, the inhibition period becomes shorter. This trend is expected, as by increasing the number of photons reaching the layer, any oxygen present in the layer is used up faster. Once the threshold concentration of oxygen above which inhibition occurs is reached (the "inhibition threshold" [15]), polymerisation can continue as normal.

This trend in inhibition period may contribute to the trend in refractive index modulation at different recording intensities for the DAG composition, shown in fig. 3 . Inhibition periods have a significant effect on the effective recording intensity. As shown in fig. 
6 , the inhibition periods are of longer duration at lower recording intensities. This will result in reduced effective recording intensities. Due to the inhibition period, a smaller number of free radicals are created initially, and thus the polymerisation occurs at a slower rate. The effect is more pronounced at the lower recording intensities. Since it leads to a lower polymerisation rate and thus more favorable ratio of the diffusion and polymerisation rates, it will ultimately lead to higher refractive index modulation, as observed in Fig. 3. As described in section 4B, this reduced rate of polymerisation is favourable for the larger diacetone acrylamide molecules, resulting in higher refractive index modulation values. As the recording intensity is increased, the inhibition period is reduced, and so the rate of polymerisation is no longer restricted in this manner.

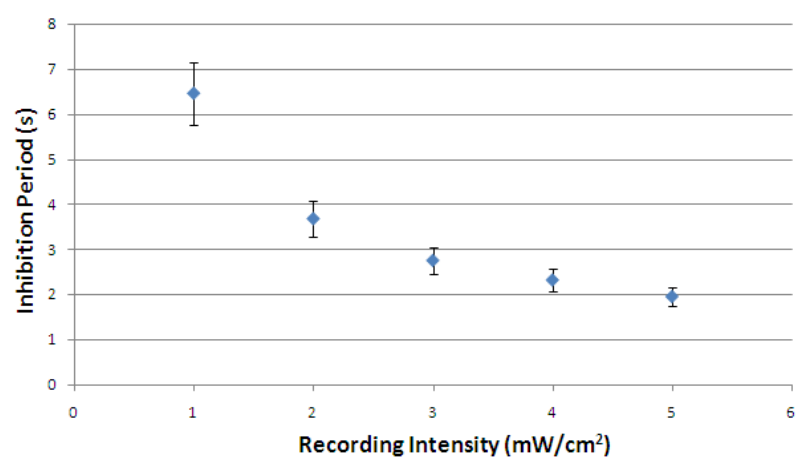

Fig. 6: Inhibition period at beginning of recording vs. recording intensity for DAG samples.

\section{E. Effect of Glycerol on the Rate of Photobleaching}

Studies on the bleaching rates of the different compositions were carried out, in order to determine if the drop observed by Meyer et al. [19] and Galassi [20] in the bleaching rate due to the presence of glycerol occurred for the new photopolymer system. In order to determine the bleaching rate, each sample was illuminated with a single $2 \mathrm{~mW} / \mathrm{cm}^{2} 532 \mathrm{~nm}$ beam. The intensity of the transmitted beam was plotted in real-time. The slope of this curve was then plotted against layer thickness, in order to obtain a picture of the dependence of the rate of bleaching on layer thickness. This was done for the DAO, DAG and AA compositions.

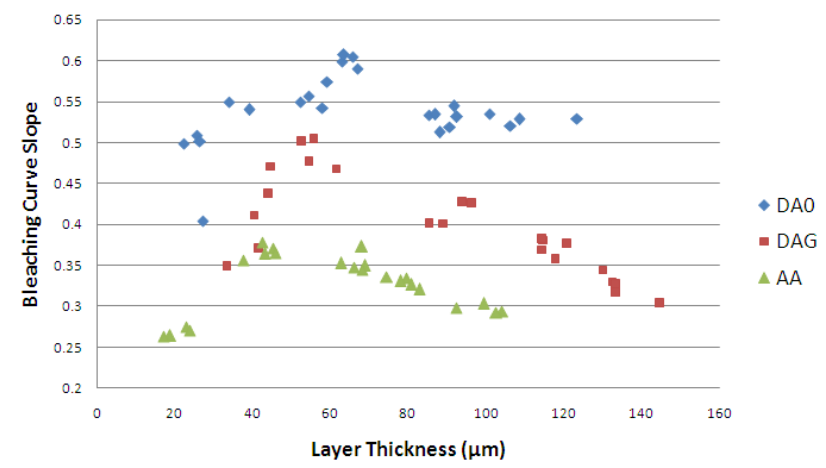

Fig. 7: Graph showing the rate of bleaching vs. layer thickness for the DAO (no glycerol), DAG (with glycerol) and AA compositions. 
In fig. 7 it is shown that the rate of photobleaching is faster for DAO than for DAG at all thicknesses. This implies that the addition of glycerol decreases the rate of photobleaching of the DA composition. As described in section 2, glycerol is a reducing agent and has been shown to stimulate the level of oxygenation in different materials. Most of the oxygen in the layer must be used up during holographic exposure before polymerisation can begin. The increased oxygen in the samples due to the presence of glycerol would inhibit the bleaching of the material via the oxygen-quenching reaction described in section 2 , which could explain the drop in bleaching rate. The AA-based photopolymer has a lower rate of photobleaching than either of the DA compositions, however this composition contains a different monomer and so the bleaching mechanisms are not directly comparable. Lower photobleaching is a desirable feature for holographic photopolymers. Slower bleaching means that more dye molecules are available to absorb a photon and initiate polymerisation, allowing for higher refractive index modulation values to be reached.

Meyer et al. showed that the bleaching rate of a glycerol-sucrose solution is dependent on its inverse viscosity [19]. Research is currently underway to investigate the interaction of Glycerol and photosensitive dyes, including Erythrosin B, by studying the dependence of the rate of photobleaching on solution viscosity. Further experiments will be carried out to compare the rate of photobleaching of the AA-based photopolymer, with and without glycerol.

\section{Conclusion}

The effect of the addition of glycerol to a new non-toxic holographic photopolymer material has been discussed. Glycerol is shown to influence the maximum achievable refractive index modulation for the new non-toxic diacetone acrylamide photopolymer, with a lower optimum recording intensity of $0.5 \mathrm{~mW} / \mathrm{cm}^{2}$ being observed. This is most likely due an increased rate of diffusion at the lower recording intensity. The addition of glycerol has also shown to reduce the rate of photobleaching of the photopolymer layers. This is thought to be due to increased levels of oxygen, and hence a higher level of oxygen quenching within the photopolymer layers during recording. Glycerol has also shown to improve the stability and optical quality of the photopolymer layers. Further characterization of the new material's holographic recording capabilities in the reflection mode is currently being carried out.

\section{Acknowledgements}

The authors acknowledge the Irish Research Council Embark Initiative for financial support, and also thank the FOCAS Research Institute for providing the research facilities to carry out the experimental work.

1. D. Cody, I. Naydenova, E. Mihaylova, "New non-toxic holographic photopolymer," J. Opt. 14, 015601 (2012).

2. S. Martin, P. Leclere, Y. Renotte, V. Toal, Y. Lion, "Characterisation of an acrylamide-based dry photopolymer holographic recording material," Opt. Eng. 33, 3942-3946 (1994).

3. D. D. McCollister, F. Oyen, V. K. Rowe, "Toxicology of Acrylamide," Toxicol. Appl. Pharm. 6, 172-181 (1964).

4. A. G. Lawrence, R. Gentry, T. McDonald, H. Bartow, J. Bounds, N. Macdonald, H. Clewell, B. Allen, C. Van Landingham, "Acrylamide: Review of Toxicity Data and Dose-Response Analyses for Cancer and Noncancer Effects," Crit. Rev. Toxicol. 36, 481-608 (2006). 
5. D. J. King, R. R. Noss, "Toxicity of polyacrylamide and acrylamide monomer," Rev. Environ. Health 8, 3-16 (1989).

6. Health implications of acrylamide in food: report of a joint FAO/WHO consultation, WHO Headquarters, Geneva, Switzerland, 25-27 June, 2002.

7. J. Siemiatycki, L. Richardson, K. Straif, B. Latreille, R. Lakhani, S. Campbell, M Rousseau and P. Boffetta, "Listing Occupational Carcinogens," Environ. Health Perspect. 112, 1447-1459 (2004).

8. Acrylamide; Sigma-Aldrich; http://www.sigmaaldrich.com/catalog/DisplayMSDSContent.do.

9. Diacetone Acrylamide; Sigma-Aldrich;

http://www.sigmaaldrich.com/catalog/DisplayMSDSContent.do.

10. M. Ortuno, E. Fernandez, S. Gallego, A. Belendez and I. Pascual, "New photopolymer holographic recording material with sustainable design," Opt. Express 15, 12425-12435 (2007).

11. S. Gallego, A. Marquez, M. Ortuno, S. Marini, J. Frances, "High environmental compatibility photopolymers compared to PVA/AA based materials at zero spatial frequency limit," Opt. Mat. 33, 531-537 (2011).

12. A. Olivares-Perez, M.P. Hernandez-Garnay, I. Fuentes-Tapia, J.C. Ibarra-Torres, "Holograms in polyvinyl alcohol photosensitized with $\mathrm{CuCl}_{2}\left(\mathrm{H}_{2} \mathrm{O}\right)$," Opt. Eng. 50, 0658011-0658016 (2011).

13. D. M. Sanderson, "A note on glycerol formal as a solvent in toxicity testing," J. Pharm. Pharmacol. 11, 150-156 (1959).

14. R. Jallapuram, I. Naydenova, S. Martin, R. Howard, V. Toal, S. Frohmann, S. Orlic, H.J. Eichler, "Acrylamide-based photopolymer for microholographic data storage," Opt. Mat. 28, 1329-33 (2006).

15. A.V. Galstyan, R.S. Hakobyan, S. Harbour, T. Galstian, "Study of the inhibition period prior to the holographic grating formation in liquid crystal photopolymerizable material,"

http://www.e-lc.org/docs/2004_05_05_11_13_17.

16. K. Pavani, "Holographic Liquid Crystal Devices," Dublin Institute of Technology, 2009.

17. Ren $X$, Yang $Z$, Kuang $T$, "Solvent-induced changes in photochemical activity and

conformation of photosystem 1 particles by glycerol," Biol. Chem. 387, 23-29 (2006).

18. C. W. Bennett, "Glycerol as Sensitizer," J. Phys. Chem. 16, 614-615 (1912).

19. T. Meyer, G. Tollin, J. Hazzard, M. Cusanovich, "Photoactive yellow protein from the purple phototropic bacterium," Biophys. J. 56, 559-564 (1989).

20. L. Galassi, "Wavelength dependence of the time course of fluorescence enhancement and photobleaching during irradiation of ethidium bromide-stained nuclei," Eur. J. Histochem. 44, 419-432 (2000).

21. H. Kogelnik, "Couple wave theory for thick hologram gratings", Bell Syst. Tech. J. 48, 29092947 (1969).

22. I. Naydenova, R. Jallapuram, R. Howard, S. Martin, V. Toal, "Investigation of the diffusion processes in a self-processing acrylamide-based photopolymer system," Appl. Opt. 43, 29002905 (2004). 